\title{
What about COVID-19 and arachidonic acid pathway?
}

\author{
Malvina Hoxha ${ }^{1}$ (D)
}

Received: 22 May 2020 / Accepted: 17 June 2020 / Published online: 25 June 2020

(C) Springer-Verlag GmbH Germany, part of Springer Nature 2020

\begin{abstract}
Background and objective COVID-19 is a highly contagious viral disease. In this study, we tried to define and discuss all the findings on the potential association between arachidonic acid (AA) pathway and COVID-19 pathophysiology.

Methods A literature search across PubMed, Scopus, Embase, and Cochrane database was conducted. A total of 25 studies were identified.

Results The data elucidated that COX-2 and prostaglandins (PGs), particularly $\mathrm{PGE}_{2}$, have pro-inflammatory action in COVID19 pathophysiology. Arachidonic acid can act as endogenous antiviral compound. A deficiency in AA can make humans more susceptible to COVID-19. Targeting these pro-inflammatory mediators may help in decreasing the mortality and morbidity rate in COVID-19 patients.

Conclusions $\mathrm{PGE}_{2}$ levels and other PGs levels should be measured in patients with COVID-19. Lowering the PGE 2 levels through inhibition of human microsomal prostaglandin E synthase-1 (mPGES-1) can enhance the host immune response against COVID-19. In addition, the hybrid compounds, such as COX-2 inhibitors/TP antagonists, can be an innovative treatment to control the overall balance between AA mediators in patients with COVID-19.
\end{abstract}

Keywords Arachidonic acid $\cdot$ COVID-19 $\cdot$ Prostaglandins $\cdot$ Leukotrienes $\cdot$ Eicosanoids $\cdot$ Thromboxane

\section{Introduction}

COVID-19 or severe acute respiratory syndrome coronavirus 2 (SARS-COV-2) is a highly contagious viral disease. Recently (April 30, 2020) we carried out a literature search for all published material on COVID 19 and arachidonic acid (AA) pathway. We searched PubMed, Scopus, Embase, and Cochrane databases using different relevant key words to identify all studies that address the association between COVID-19 and AA pathway. The following text words were used: "COVID19 and prostaglandin," "COVID19 and thromboxane," "COVID19 and leukotriene," "COVID19 and lipoxin," "COVID19 and 5-lipoxygenase," "COVID19 and 12-lipoxygenase," "COVID19 and 15-lipoxygenase," "COVID19 and cytochrome P450 epoxygenase pathway," "COVID19 and pro resolving lipid mediators," and "COVID19 and NSAIDs." Twenty-five studies were initially

Malvina Hoxha

m.hoxha@unizkm.al

1 Department of Chemical-Toxicological and Pharmacological Evaluations of Drugs, Faculty of Pharmacy, Catholic University Our Lady of Good Counsel, Rruga Dritan Hoxha, Tirana, Albania identified. The purpose of this literature review is to synthesize the available information on the AA pathway involvement in COVID-19.

Arachidonic acid is a polyunsaturated fatty acid produced by membrane phospholipids through phospholipase- $\mathrm{A}_{2}$ $\left(\mathrm{PLA}_{2}\right)$ in inflammatory condition. Das et al. suggested that AA can act as endogenous antiviral compound and can contribute to the inactivation of enveloped viruses, such as influenza virus, HIV, or SARS-CoV-2 [1]. The capacity to induce leakage or lysis of microbial cell membranes, to inhibit the respiratory activity, to uncouple the oxidative phosphorylation are some of the mechanisms responsible for the antimicrobial action of AA [1, 2]. Consequently, Das et al. suggested that $\mathrm{T}$ and $B$ cells, leukocytes, macrophages, and other cells release AA when affected by viruses, such as SARS-CoV-2, and can inactivate the invading microorganisms [1]. Hence, a deficiency in AA can make humans more susceptible to SARS-CoV-2 [1].

AA can be a substrate for different pathways, such as cyclooxygenase (COX) and lipooxygenase (LOX) pathway, and can give rise to different mediators, which control inflammation. Prostaglandins $(\mathrm{PG})$ and thromboxane $\left(\mathrm{TXA}_{2}\right)$ are proinflammatory lipid mediators produced through COX pathway. LOX gives rise to leukotrienes and lipoxins which 
possess pro- and anti-inflammatory activities, respectively. In contrast with these pro-inflammatory lipid mediators, other AA mediators, such as lipoxins, resolve inflammation and have potent anti-inflammatory activity [1]. There are different types of $\mathrm{PGs}\left(\mathrm{PGE}_{2}, \mathrm{PGI}_{2}, \mathrm{PGD}_{2}, \mathrm{PGF}_{2 \alpha}\right)$ which contribute to the inflammatory process.

Following viral infection, an activation of immune response occurs, which includes the release of inflammatory mediators, such as pro-inflammatory cytokines (IL-6, IL-1 $\beta, \mathrm{TNF}-\alpha$ ) and eicosanoids (prostaglandins and leukotrienes) [3]. The second isoform of cyclooxygenase (COX-2) is responsible for producing the major part of PGs that are responsible for pain and inflammation [4]. Analogously, the SARS coronavirus responsible for the 2003 outbreak increased the production of PGs by binding to COX-2 [4]. Different studies have demonstrated that $\mathrm{PGE}_{2}$ increases the viral pathogenicity in many viral infections such as CMV, RB, RSV, HSV, EV71, and CVB2 by interfering with the viral transcription translation and/or replication [3]. However, we should also consider that in some cases, such as in HBV and PIV3, $\mathrm{PGE}_{2}$ can either stimulate or inhibit the viral pathogenicity [3]. Aso et al. reported that in human pulmonary microvascular endothelial cells, $\mathrm{PGE}_{2}$ can play an upstream essential role in inflammation, leading to the increase of COX-2 expression, without affecting the COX-1 isoform, and increasing IL-8 [5].

Smeitink et al. suggested that $\mathrm{PGE}_{2}$ has a significant role in COVID-19 pathophysiology hyperinflammatory and immune responses [3]; therefore, $\mathrm{PGE}_{2}$ can be measured in patients with COVID-19. The same author also hypothyze that lowering the $\mathrm{PGE}_{2}$ levels through inhibition of human microsomal prostaglandin E synthase-1 (mPGES-1) can enhance the host immune response against COVID-19 and moreover can be a promising therapeutic strategy for preventing severe disease progression and death. Moreover, mPGES-1 inhibition has the advantage of not effecting other PGs level and of allowing basal biosynthesis of $\mathrm{PGE}_{2}$ by the two other constitutive synthases (cPGES and mPGES-2). Selective inhibition of mPGES-1 has been suggested as a therapeutic alternative to stimulate antiviral immunity in mice with influenza A virus (IAV). mPGES-1 inhibitors increased the survival of IAV mice [6]. Sonlicromanol (KH176; IUPAC chemical name (S)-6-hydroxy-2,5,7,8-tetramethyl-N-((R)piperidin-3yl)chroman-2-carboxamide hydrochloride) is a selective inhibitor of mPGES-1 that is currently under phase 2 b study [7]. Sonlicromanol can attenuate the side effects of COX inhibitors.

In respect to gender differences, Pace et al. observed that in humans during acute inflammation, there was an increased expression of COX-2 and $\mathrm{PGE}_{2}$ in males vs females [8]. Following these findings, it is possible that the increased $\mathrm{PGE}_{2}$ levels in males can be one of the underline factors contributing to a more severe COVID-19 infection in males [3].
Moreover, other studies conducted in animals have reported that $\mathrm{PGE}_{2}$ levels were affected by age [9], which can also be an underline factor of the hypersensitivity of older patients in respect to children with COVID-19 [3]. Considering that COVID-19 patients with comorbidities, such as obesity, have a higher risk of disease aggravation, $\mathrm{PGE}_{2}$ may also contribute to the severity of the disease in these patients, considering that different studies have reported an increase of $\mathrm{PGE}_{2}$ in obese patients [10]. In addition, $\mathrm{PGE}_{2}$ can also contribute to intravascular thrombosis [11], which is another complication present in patients with COVID-19.

Isoprostanes are generated in vivo by free radical attack of AA. Reactive oxygen species (ROS) were found in plasma and lung tissue in patients with acute respiratory distress syndrome (ARDS), and the mPGES-1 selective inhibitor, sonlicromanol, showed radical scavenging activity and prevented ROS-driven cell death [12].

$\mathrm{PGD}_{2}$ is another prostaglandin of interest for respiratory viruses. In an animal experimental model, infected with respiratory viruses, there was a higher production of $\mathrm{PGD}_{2}$, which was further increased in older mice [13]. Vijay et al. suggested that $\mathrm{PGD}_{2}$ has an important role in the lung inflammatory state by regulating different steps in the $\mathrm{T}$ cells responses in mice with SARS-CoV and MERS-CoV [13]. In addition, Werder et al. reported that $\mathrm{PGD}_{2}$ production was increased in patients with severe syncytial virus (RSV) bronchiolitis, suggesting that DP agonists may be useful antivirals for the treatment of viral bronchiolitis and possibly as primary preventatives for asthma [14]. Moreover, 15d-PGJ $\mathrm{d}_{2}$ a $\mathrm{PGD}_{2}$ derivative, which acts through DP1 receptor, has shown to reduce influenza morbidity and mortality in mice through PPAR $\gamma$ pathway [15]. Based on these findings, it would be of interest to measure the $\mathrm{PGD}_{2}$ levels in COVID-19 patients.

\section{$\mathrm{PLA}_{2}$}

Phospholipase $\mathrm{A}_{2}$ group IID (PLA $\mathrm{P}_{2} \mathrm{G} 2 \mathrm{D}$ ) belongs to the phospholipases family and shows age-dependent increases in the lungs. Zheng et al. hypothyzed that PLA $\mathrm{A}_{2} \mathrm{D}$ deficiency in myeloid cells plays an essential role in the formation of CD4 Tfh cells and humoral immune memory against respiratory coronavirus infection [16]. PLA $\mathrm{PLX}_{2}$ pression is also increased in $\mathrm{HCV}$ virus and is involved in $\mathrm{HCV}$ virus replication, making it a potential therapeutic target for anti-HCV therapy [17]. Other data suggested that CM-II-sPLA 2 is a potential alternative for the development of broad-spectrum antiviral drugs that target viral envelope lipid bilayers derived from the endoplasmic reticulum membrane [18]. Currently there are no data on the role of $\mathrm{PLA}_{2}$ in COVID-19, and measuring its levels in these patients would not only fill this gap of knowledge but can give us further information on new therapeutic targets. 


\section{LOX pathway}

Other than COX-2 and $\mathrm{PGE}_{2}$, also 5-LOX pathway is involved in virus pathophysiology. Leukotrienes, especially $\mathrm{LTB}_{4}$, inhibit influenza viral replication in vivo [19]. Influenza virus upregulates 5 -LO in lungs of either infected animal models (mice) or humans [20]. $\mathrm{LTB}_{4}$-treated neutrophils had enhanced virucidal activity against influenza virus, human coronavirus, and RSV [21]. In addition, higher levels of cysLTs observed in mice with influenza virus increased the survival rate [22]. Currently we did not identify any study on the implication of leukotrienes in COVID-19.

\section{NSAIDs and COXIBs}

NSAIDs block the cyclooxygenase enzymes (COX-1/COX2 ), which are responsible for the production of inflammatory mediators (PG, LT, TX). Fang et al., while studying the severity of COVID-19 symptoms in patients with asthma and hypertension, linked the SARS-CoV-2 to the downregulation of angiotensin-converting enzyme-2 (ACE2), which instead is upregulated by ibuprofen [23]. Amici et al. reported that indomethacin, which is generally prescribed for treatment of gout and arthritis, inhibited human virus SARS-CoV replication at a concentration dose of $1 \mathrm{mg} / \mathrm{kg}$ [24]. However, different studies have questioned the role of NSAIDs and specifically of ibuprofen in patients with COVID-19. The reason behind that is that NSAIDs use in long term has been associated with several side effects, such as gastrointestinal and cardiovascular complications, nephrotoxicity, etc. Voiriot et al. reported that after respiratory tract infection, NSAIDs were associated with severe complications, such as respiratory tract infection and peritonsillar abscess [25]. NSAIDs inhibit COX; hence, they also inhibit the synthesis of antiinflammatory AA mediators, specifically, lipoxins and resolvins, which lead to a delay of the resolution of the inflammation [25]. In line with this, Basille et al. showed that despite NSAIDs were taken for long term or for short term to treat acute illness, they were associated with respiratory complications [26].

But does this evidence also apply to COVID-19 pandemic? On March 2020, the French health authorities published a warning on the severe side effects of NSAIDs in patients with COVID-19 [27]. EMA has declared no evidence of NSAIDs and ibuprofen in COVID-19 worsening [28]. In line with this, the UK Medicines and Healthcare Products Regulatory Agency (MHRA) reported that there is not a current research on ibuprofen and COVID-19 worsening [29]. Moreover, WHO suggests the use of ibuprofen as antipyretic drug and paracetamol as a first treatment option for fever or pain. Patients that take NSAIDs for different reason should not stop using them for fear of their COVID-19 risks [4].
Considering that NSAIDs can cause gastric side effects (ulcer, bleeding) or an increased risk of cardiovascular problems, which can be explained by a disbalance between AA mediators (prostacyclin and TX), the selective inhibition of mPGES- 1 can be a better strategy in controlling inflammation in COVID-19. Das et al. suggested that also an intravenous or oral administration of AA can help in increasing the resistance, facilitating the recovery, or even preventing (higher AA levels) SARS-CoV-2 [1]. In addition we also hypothyze that dual compounds COX-2 inhibitors/TP antagonist, providing the anti-inflammatory effect and cardiovascular safety, may be an effective strategy in patients with SARS-CoV-2.

Concluding, we must not underestimate the importance of AA mediators in promoting or controlling inflammation in COVID-19; therefore, we believe that further studies on AA pathway implication in COVID-19 can contribute in developing new therapeutic strategies combating inflammation.

Author's contributions M.H is the only author of the manuscript who performed the literature search, drafted the manuscript, and reviewed the manuscript for its intellectual content.

\section{Compliance with ethical standards}

Conflict of interest The author declares that has no conflict of interest.

\section{References}

1. Das U (2020) Can bioactive lipids inactivate coronavirus (COVID19)? Arch Med Res. [Google Scholar]

2. Das U (2018) Arachidonic acid and other unsaturated fatty acids and some of their metabolites function as endogenous antimicrobial molecules: a review. J Adv Res 11:57e66 Google Scholar

3. Smeitink J, Jiang X, Pecheritsyna S, Renkema H, Van Maanen R, Beyrath J (2020) Hypothesis: mPGES-1-derived prostaglandin E2, a so far missing link in COVID-19 pathophysiology?. Preprints, 2020040180 (doi: https://doi.org/10.20944/preprints202004.0180. v1). [Google Scholar]

4. FitzGerald GA (2020) Misguided drug advice for COVID-19. Science 367(6485):1434 Google Scholar

5. Aso H, Ito S, Mori A, Morioka M, Suganuma N, Kondo M et al (2012) Prostaglandin e 2 enhances interleukin-8 production via ep4 receptor in human pulmonary microvascular endothelial cells. Am J Phys Lung Cell Mol Phys 302:266-273 Google Scholar

6. Park JH, Park EB, Lee JY, Min JY (2016) Identification of novel membrane-associated prostaglandin e synthase-1 (mPGES-1) inhibitors with anti-influenza activities in vitro. Biochem Biophys Res Commun 469:848-855 Google Scholar

7. Janssen MCH, Koene S, de Laat P, Hemelaar P, Pickkers P, Spaans E, et al. (2019) The KHENERGY study: safety and efficacy of KH176 in mitochondrial m.3243A $>$ G spectrum disorders. Clin Pharmacol Ther . [Google Scholar]

8. Pace S, Rossi A, Krauth V, Dehm F, Troisi F, Bilancia R et al (2017) Sex differences in prostaglandin biosynthesis in neutrophils during acute inflammation. Sci Rep 7:1-10 Google Scholar

9. Wu D, Mura C, Beharka AA, Han SN, Paulson KE, Hwang D, et al. (1998) Age-associated increase in PGE2 synthesis and COX 
activity in murine macrophages is reversed by vitamin $\mathrm{E}$. Am J Phys Cell Phys, 275. [Google Scholar]

10. Fain JN, Kanu A, Bahouth SW, Cowan GSM, Hiler ML, Leffler CW (2002) Comparison of PGE2, prostacyclin and leptin release by human adipocytes versus explants of adipose tissue in primary culture. Prostaglandins Leukot Essent Fat Acids 67:467-473 Google Scholar

11. Gross S, Tilly P, Hentsch D, Vonesch JL, Fabre JE (2007) Vascular wall-produced prostaglandin E2 exacerbates arterial thrombosis and atherothrombosis through platelet EP3 receptors. J Exp Med 204:311-320 Google Scholar

12. Beyrath J, Pellegrini M, Renkema H, Houben L, Pecheritsyna S, Van Zandvoort P, et al. (2018) KH176 safeguards mitochondrial diseased cells from redox stress-induced cell death by interacting with the thioredoxin system/peroxiredoxin enzyme machinery. Sci Rep. [Google Scholar]

13. Vijay R, Fehr AR, Janowski AM, Athmer J, Wheeler DL, Grunewald M et al (2017) Virus-induced inflammasome activation is suppressed by prostaglandin D2/DP1 signaling. Proc Natl Acad Sci 114(27):E5444-E5453 Google Scholar

14. Werder R, Lynch J, Simpson J, Zhang V, Hodge N, Poh M, Blom EF, Kulis Ch, Smythe M, Upham J, et al. (2018) PGD2/DP2 receptor activation promotes severe viral bronchiolitis by suppressing IFN- $\lambda$ production. Sci Transl Med. 10(440). [Google Scholar]

15. Cloutier A, Marois I, Cloutier D, Verreault C, Cantin A, Richter M (2012) The prostanoid 15-Deoxy- $\Delta^{12,14}$-prostaglandin- $J_{2}$ reduces lung inflammation and protects mice against lethal influenza infection. J Infect Dis 205(4):621-630 Google Scholar

16. Zheng J, Perlman S (2019) Phospholipase A2 group IID is indispensable for coronavirus-specific humoral immune memory in middle aged mice. J Immunol, 202 (1 Supplement) 140.13. [Google Scholar]

17. Xu S, Pei R, Guo M, Han Q, Lai J, Wang Y, Wu C, Zhou Y, Lu M, Chen X (2012) Cytosolic phospholipase A2 gamma is involved in hepatitis C virus replication and assembly. J Virol 86(23):1302513037 Google Scholar

18. Chen M, Aoki-Utsubo C, Kameoka M et al (2017) Broad-spectrum antiviral agents: secreted phospholipase $\mathrm{A}_{2}$ targets viral envelope lipid bilayers derived from the endoplasmic reticulum membrane. Sci Rep 7:15931 Google Scholar

19. Gaudreault E, Gosselin J (2008) Leukotriene B4 induces release of antimicrobial peptides in lungs of virally infected mice. J Immunol 180(9):6211-6221 Google Scholar
20. Gentile DA, Fireman P, Skoner DP (2003) Elevations of local leukotriene C4 levels during viral upper respiratory tract infections. Ann Allergy Asthma Immunol 91(3):270-274 Google Scholar

21. Widegren H, Andersson M, Borgeat P, Flamand L, Johnston S, Greiff L (2011) LTB4 increases nasal neutrophil activity and conditions neutrophils to exert antiviral effects. Respir Med 105(7): 997-1006 Google Scholar

22. Carey MA, Bradbury JA, Seubert JM, Langenbach R, Zeldin DC, Germolec DR (2005) Contrasting effects of cyclooxygenase-1 (COX-1) and COX-2 deficiency on the host response to influenza A viral infection. J Immunol 175(10):6878-6884 Google Scholar

23. Fang L, Karakiulakis G, Roth M (2020) Are patients with hypertension and diabetes mellitus at increased risk for COVID-19 infection? Lancet Respir Med. https://doi.org/10.1016/S2213-2600(20) 30116-8

24. Amici C, Di Caro A, Ciucci A et al (2006) Indomethacin has a potent antiviral activity against SARS coronavirus. Antivir Ther 11(8):1021-1030

25. Voiriot G, Philippot Q, Elabbadi A, Elbim C, Chalumeau M, Fartoukh M (2019) Risks related to the use of non-steroidal antiinflammatory drugs in community-acquired pneumonia in adult and pediatric patients. J Clin Med 8:E786. https://doi.org/10. 3390/jcm806078631163625

26. Basille D, Thomsen RW, Madsen M et al (2018) Nonsteroidal antiinflammatory drug use and clinical outcomes of communityacquired pneumonia. Am J Respir Crit Care Med 198:128-131. https://doi.org/10.1164/rccm.201802-0229LE29461860

27. Willsher K (2020) Antiinflammatories may aggravate COVID19 France Advises "The Guardian". [Google Scholar]

28. European Medicines agency. EMA gives advice on the use of nonsteroidal anti- inflammatories for COVID-19 [online]. 2020. Available: https://www.ema.europa.eu/en/news/ema-gives-adviceuse-non-steroidal-anti-inflammatories-covid-19 [Accessed 24 March 2020

29. Medicines and healthcare products regulatory agency. ibuprofen use and Coronavirus (COVID-19) [online]. 2020. Available: https://www.gov.uk/government/news/ibuprofen-use-andcovid19coronavirus [accessed 24 March 2020]. [Google Scholar]

Publisher's note Springer Nature remains neutral with regard to jurisdictional claims in published maps and institutional affiliations. 